\title{
Information Capacity of Vesicle Release in Neuro-spike Communication
}

\author{
Hamideh Ramezani ${ }^{1}$, Student Member, IEEE and Ozgur B. Akan ${ }^{2}$, Fellow, IEEE
}

\begin{abstract}
Information transmission in the nervous system is performed through propagation of spikes among neurons, which is done by vesicle release to chemical synapses. Understanding the fundamentals of this communication can lead to the implementation of bio-inspired nanoscale communication paradigms. In this paper, we utilize a realistic pool-based model for vesicle release and replenishment in hippocampal pyramidal neurons and evaluate the capacity of information transmission in this process by modeling it as a binary channel with memory. Then, we derive a recurrence relation for the number of available vesicles, which is used to find successful bit transmission probabilities and mutual information between input and output. Finally, we evaluate the spiking probability that maximizes mutual information and derive the capacity of the channel.
\end{abstract}

Index Terms-Molecular communication, neuro-spike communication, nanonetworks, vesicle release process, channel capacity.

\section{INTRODUCTION}

$\mathbf{N}$ EURO-SPIKE communication is a significant example of molecular communication among nanomachines. In this communication, information is encoded in spike trains and transmitted through neural pathways by release of vesicles that contain neurotransmitters to the gap between neurons [1]. Several communication channel models are introduced for each of the biological processes involved in this communication [2][6] and the characteristics of these communication channels are investigated under different assumptions [3]-[8]. However, the impact of the number of available vesicles for release on its capacity is not evaluated in the existing studies [6]-[8].

A wide range of models are used in the literature to investigate the stochastic nature of vesicle release, from using a fixed probability distribution [4] to utilizing finite state markov channels [3] and pool-based models [6], [9]. Among them, a realistic pool-based release and refill model provides insight on how the availability of vesicles for release affects the capacity of this communication. Although a pool-based release model is considered in [6], the number of available vesicles are overestimated since the refill rate is assumed to be proportional to the number of reserve vesicles. In reality, the replenishment is much slower since released vesicles are refilled by the vesicles that are nearer to the release site [9].

In this paper, we use a realistic pool-based model for vesicle release and replenishment in hippocampal pyramidal neurons

\footnotetext{
${ }^{1} \mathrm{H}$. Ramezani is with the Next-generation and Wireless Communications Laboratory (NWCL), Department of Electrical and Electronics Engineering, Koc University, Istanbul, Turkey hramezani13@ku.edu.tr

${ }^{2} \mathrm{O}$. B. Akan is with the Internet of Everything (IoE) Group, Electrical Engineering Division, Department of Engineering, University of Cambridge, UK oba21@cam.ac.uk
}

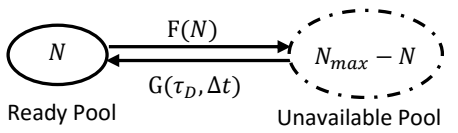

Fig. 1. Pool-based model for neurotransmitter release and replenishment.

and derive the binary channel model for vesicle release process by discretizing the time. Then, we derive the successful bit transmission probabilities and calculate the mutual information between input and output of the channel. Finally, we evaluate the capacity of vesicle release process.

The remainder of this paper is organized as follows. In Section II, the biological background of vesicle release process and its realistic pool-based model are presented. The mutual information between input and output of the channel, the optimum spiking probability to reach the maximum mutual information and the channel capacity are derived in Section III. Then, simulations results are reported in Section IV to evaluate effects of availability of vesicles on capacity of the channel. Finally the paper is concluded in Section V.

\section{MOdel Description}

Arrival of the spike to the axonal terminal changes the membrane potential of the neuron, which in turn opens the voltage dependent calcium channels (VDCCs) and allows entering of calcium ions to the neuron. The increment in concentration of $\mathrm{Ca}^{2+}$ near the vesicles that are ready to be released initiates the fusion of these vesicles to the membrane, causing the release of their contents to the synapse, i.e., the gap between neurons [10]. Based on [9], vesicles are grouped into at least two distinct pools, a pool of readily releasable vesicles (RRVs) called ready pool (RP), and a larger pool containing the vesicles farther from the pre-synaptic site. Each spike can initiate the release of at most one RRV from each pre-synaptic terminal in hippocampal pyramidal neurons [11]. Hence, we consider the univesicular release model and use the single-pool model depicted in Fig. 1 to properly model the vesicle release and replenishment in these neurons [9]. In Fig. $1, N_{\max }$ is the capacity of RP, i.e., the number of RRVs when all of them are recovered, $F(N)$ is the release probability when the RP has $N$ vesicles with $N \leq N_{\max }$, $\tau_{D}$ is the mean recovery time of a vacancy, and $G\left(\tau_{D}, \Delta t\right)$ is the probability of one vacancy replenishment after $\Delta t$ second. Since the number of vesicles ready to be docked in neurons is much higher than $N_{\max }$ [9], the recovery of a vacancy can be modeled by the first event from a Poisson process [12]. Hence, $G\left(\tau_{D}, \Delta t\right)=1-\exp \left(-\tau_{D}^{-1} \Delta t\right)$, and vesicle recovery 
after $\Delta t$ second is derived based on Binomial distribution as $B\left(N_{\max }-N, G\left(\tau_{D}, \Delta t\right)\right)$.

To find the vesicle release probability, we study both spontaneous and evoked release scenarios in the following.

a) Evoked Release: Prior to release of any vesicle, the release of each RRV as a result of the spike arrival is independent from others and is governed by a Poisson process with rate $\lambda_{v}(t)$. This rate, $\lambda_{v}(t)$, depends on (i) opening of VDCCs and their distance from calcium sensors, (ii) binding of calcium ions to buffers, calcium sensors and pumps, and (iii) diffusion of calcium ions and buffers. Considering the start time of spike arrival as $t_{0}$ and the spike duration as $\Delta t_{s}$, the evoked release also happens in the time interval given by $\left[t_{0}, t_{0}+\Delta t_{s}\right]$. The release or fusion rate for a single vesicle during this interval is then defined as $\alpha_{v}(\Delta t)=\int_{t_{0}}^{t_{0}+\Delta t} \lambda_{v}(t) d t$, where $\Delta t \leq \Delta t_{s}$. In hippocampal pyramidal neurons, release of one of the vesicles transiently prevents fusion of others with membrane [9]. Hence, the probability of evoked release in the time interval given by $\left[t_{0}, t_{0}+\Delta t\right]$ when RP contains $N$ vesicles is derived as $\left\{\begin{array}{ll}1-\exp \left(-N \alpha_{v}(\Delta t)\right), & \Delta t \leq \Delta t_{s} \\ 1-\exp \left(-N \alpha_{v}\left(\Delta t_{s}\right)\right), & \Delta t>\Delta t_{s}\end{array}[6]\right.$.

b) Spontaneous Release: According to recordings from Hippocampal neurons [13], the average waiting time for spontaneous release of each RRV is eight minutes. Hence, the spontaneous release probability from a synapse with $N$ vesicles during $\Delta t$ second is derived as $1-\exp \left(-\frac{N \Delta t}{480}\right)$.

\section{CAPACITY ANALYSIS}

By discretizing time into windows of equal durations, i.e., $\Delta t$, and selecting $\Delta t$ sufficiently small, at most one spike can exist at each window [6]. Note that selecting $\Delta t<\Delta t_{s}$ makes the vesicle release at $\frac{\Delta t_{s}}{\Delta t}$ consecutive windows dependent on each other as a result of the univesicular release model. Hence, it complicates the system model while not affecting the final results since during each $\Delta t_{s}$ second only one spike, thus one vesicle release, can occur in real scenario. Hence, we select $\Delta t \geq \Delta t_{s}$ to have the spike shape, which is the input of vesicle release process, in one window. Thus, vesicle release process can be modeled by a binary channel whose input, i.e., $S_{n}$, and output, i.e., $V_{n}$, indicate existence of spike and vesicle release in $n$th window, respectively. Successful bit transmission in each window depends on the number of RRVs and vesicle release probability as given below.

$$
\begin{aligned}
T_{11}(n) & \triangleq P\left\{V_{n}=1 \mid S_{n}=1\right\} \\
& =\sum_{l=0}^{N_{\max }} P\left\{V_{n}=1 \mid N_{n}=l, S_{n}=1\right\} P\left\{N_{n}=l\right\}, \\
T_{00}(n) & \triangleq P\left\{V_{n}=0 \mid S_{n}=0\right\} \\
& =\sum_{l=0}^{N_{\max }} P\left\{V_{n}=0 \mid N_{n}=l, S_{n}=0\right\} P\left\{N_{n}=l\right\},
\end{aligned}
$$

where $N_{n}$ indicates number of available vesicles in RP at the beginning of $n$th time slot. Moreover, the probability of evoked and spontaneous release in $n$th time slot when RP has $N$ vesicles are expressed as follows.

$$
\begin{aligned}
& P\left\{V_{n}=1 \mid N_{n}=N, S_{n}=1\right\}=1-\exp \left(-N \alpha_{v}\left(\Delta t_{s}\right)\right) \\
& P\left\{V_{n}=1 \mid N_{n}=N, S_{n}=0\right\}=1-\exp \left(-\frac{N \Delta t}{480}\right) .
\end{aligned}
$$

Defining $P_{n}$ as a $1 \times\left(N_{\max }+1\right)$ array with $i$ th element equal to $P\left\{N_{n}=i-1\right\}$, a recurrence relation for $P_{n}$ is derived as

$$
P_{n}=P_{n-1} D R,
$$

where $D$ and $R$ are $\left(N_{\max }+1\right) \times\left(N_{\max }+1\right)$ matrices indicating probability of changes in number of RRVs as a result of pool depletion and replenishment, respectively.

Vesicle depletion depends on arrival of spike to the axonal terminal, which can be modeled by a Poisson process [6], [7]. Considering rate of this process as $\lambda$, probability of spike arrival at $n$th slot can be calculated as $P\left\{S_{n}=1\right\}=1-$ $\exp (-\lambda \Delta t) \triangleq p$. Then, the vesicle release probability in $n$th slot when RP has $i$ vesicles, $F_{n}(i)$, is derived as follows.

$$
F_{n}(i)=1-\left[\exp \left(-i \alpha_{v}\left(\Delta t_{s}\right)\right) p+\exp \left(-\frac{i \Delta t}{480}\right)(1-p)\right]
$$

Hence, the nonzero elements of matrix $\mathrm{D}$ are derived as $D_{11}=$ $1, D_{i i}=1-F_{n}(i-1)$ and $D_{i(i-1)}=F_{n}(i-1)$ for $i>1$.

Matrix $R$ is used to find impact of vesicle replenishment on the number of available RRVs. It is an upper triangular matrix whose $(i, j)$ th element for $\forall j \geq i$ is defined as follows.

$$
R_{i j}=\left(\begin{array}{c}
N_{\max }-(i-1) \\
j-i
\end{array}\right) G\left(\tau_{D}, \Delta t\right)^{j-i}\left(1-G\left(\tau_{D}, \Delta t\right)\right)^{N_{\max }-(j-1)}
$$

Using the probabilities given in (1) and (2), the mutual information between input and output of the channel in $n$th time slot can be expressed as follows.

$$
\begin{aligned}
I\left(S_{n} ; V_{n}\right)=\mathcal{H} & \left((1-p)\left(1-T_{00}(n)\right)+p T_{11}(n)\right) \\
& -\left[(1-p) \mathcal{H}\left(T_{00}(n)\right)+p \mathcal{H}\left(T_{11}(n)\right)\right],
\end{aligned}
$$

where $\mathcal{H}(\mathcal{P})=-\mathcal{P} \log _{2} \mathcal{P}-(1-\mathcal{P}) \log _{2}(1-\mathcal{P})$. Moreover, the maximum mutual information achieved from 1st to $n$th time slot is represented as $C_{n}=\max _{p} \sum_{l=1}^{n} \frac{I\left(S_{l} ; V_{l}\right)}{n} \quad$ bit/slot. Furthermore, the channel capacity is defined as $C=\lim _{n \rightarrow \infty} C_{n}$.

To find the channel capacity, we evaluate the convergence of $I\left(S_{n} ; V_{n}\right)$. Based on the recurrent equation given by (3), the number of vesicles in RP can be modeled by a finitestate Markov chain. Moreover, the steady state probability of each state in this Markov chain, i.e., $\pi=\left[\pi_{0}, \pi_{1}, \ldots, \pi_{N_{\max }}\right]$, can be derived by solving the linear equations $\pi=\pi D R$ and $\sum_{i=0}^{N_{\max }} \pi_{i}=1$. Then, the probabilities of successful bit transmission, thus $I\left(S_{n} ; V_{n}\right)$, converge as given below.

$$
\begin{aligned}
\lim _{n \rightarrow \infty} T_{11}(n)= & \sum_{l=0}^{N_{\max }} P\left\{V_{n}=1 \mid N_{n}=l, S_{n}=1\right\} \pi_{l} \triangleq T_{1, \infty}, \\
\lim _{n \rightarrow \infty} T_{00}(n)= & \sum_{l=0}^{N_{\max }}\left(1-P\left\{V_{n}=1 \mid N_{n}=l, S_{n}=0\right\}\right) \pi_{l} \triangleq T_{0, \infty}, \\
\lim _{n \rightarrow \infty} I\left(S_{n} ; V_{n}\right)= & \mathcal{H}\left((1-p)\left(1-T_{0, \infty}\right)+p T_{1, \infty}\right) \\
& -\left[(1-p) \mathcal{H}\left(T_{0, \infty}\right)+p \mathcal{H}\left(T_{1, \infty}\right)\right] \triangleq I_{\infty} .
\end{aligned}
$$




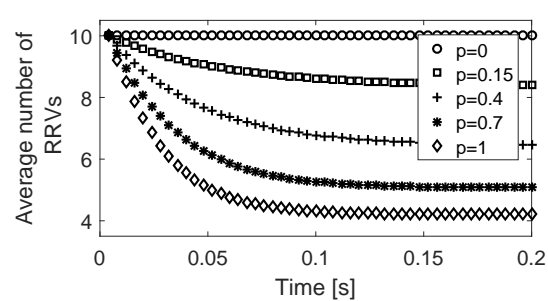

(a) $N_{\max }=10, \tau_{D}=\frac{0.6}{N_{\max }}$

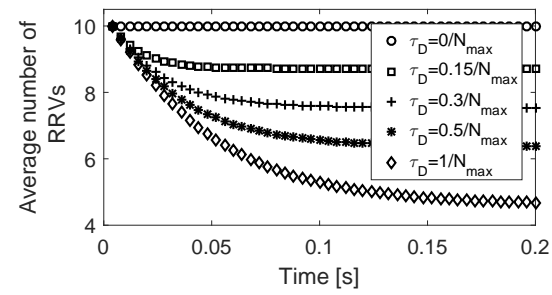

(b) $N_{\max }=10, p=0.5$

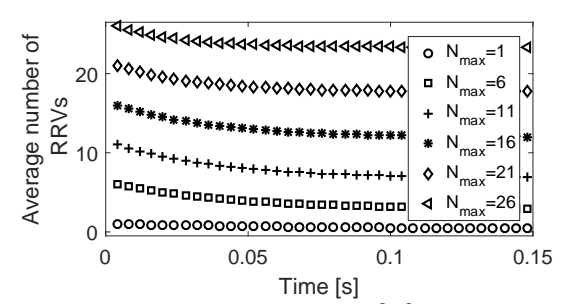

(c) $p=0.5, \tau_{D}=\frac{0.6}{N_{\max }}$

Fig. 2. Average number of RRVs for different (a) spiking probabilities, (b) mean recovery times of a vacancy, and (c) capacities of RP.

Hence, $\lim _{n \rightarrow \infty} \sum_{l=1}^{n} \frac{I\left(S_{l} ; V_{l}\right)}{n}=I_{\infty}$ and the channel capacity can be calculated as $C=\max _{p} I_{\infty}$.

\section{Evaluation}

In this section, we evaluate changes in the number of RRVs over time and their effects on mutual information between input and output of the channel. Moreover, we analyze the variations in the probability that maximizes the average mutual information and study the capacity of the channel.

The range of parameters are selected according to reports in biological studies [12], [14] and time step is set equal to width of a spike, i.e., $\Delta t=\Delta t_{s}=4 \mathrm{~ms}$ [10]. Moreover, we utilize $\alpha_{v}\left(\Delta t_{s}\right)=0.06 \sqrt{N}$ as reported in [15] for the average fusion rate upon spike arrival by using realistic assumptions for concentration of $\mathrm{Ca}^{2+}$ in pre-synaptic terminal.

\section{A. Number of Readily Releasable Vesicles}

Number of available vesicles in RP is changing over time according to the pool-based model shown in Fig. 1. Hence, characteristics of the depletion and replenishment mechanisms affect the probability of having a specific number of RRVs. Based on (3), important parameters in determining this probability are spiking probability, $p$, capacity of RP, $N_{\max }$, and mean recovery time of a vacancy, $\tau_{D}$. Effects of these parameters on the average number of RRVs are shown in Fig. 2. Since probability of spontaneous release is much less than evoked release, increasing spiking probability, $p$, causes more release of neurotransmitters in a given time based on (4), which in turn decreases the average number of RRVs as shown in Fig. 2(a). Moreover, increasing mean recovery time of a vacancy, $\tau_{D}$, slows down the vacancy replenishment process and decreases the average number of RRVs as depicted in Fig. 2(b). Hence, the exact recovery process is an important factor in analyzing the channel capacity. In remainder of this section, we utilize $\tau_{D}=\frac{0.6}{N_{\max }}$ as reported in [12].

For RPs with higher capacity, not only RP has more RRVs at beginning of communication, but also $\tau_{D}$ is smaller. Hence, average number of RRVs is higher as shown in Fig. 2(c).

\section{B. Mutual Information}

Mutual information among input and output of the channel at each slot depends on the availability of vesicles in RP. To evaluate the changes in $I\left(S_{n} ; V_{n}\right)$, its value for different slots and spiking probabilities is shown in Fig. 3, where the

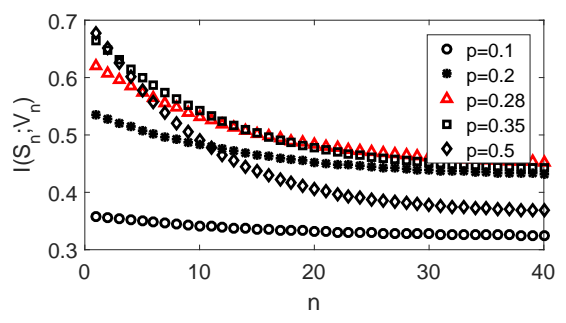

Fig. 3. Mutual information between spiking and vesicle release in $n$th slot.

capacity of the RP is set to $N_{\max }=10$. Since the average number of RRVs decreases by time, $I\left(S_{n} ; V_{n}\right)$ also reduces with time. Moreover, the reduction rate of mutual information in first slots is higher for more probable spiking as expected. Furthermore, the mutual information of the channel first increases and then decreases by increasing spiking probability, $p$. This pattern is more visible in Fig. 4(a), where the average mutual information from slot 1 to $\mathrm{n}, \sum_{l=1}^{n} \frac{I\left(S_{l} ; V_{l}\right)}{n}$, is shown for different spiking probabilities. As illustrated in Fig. 4(a), the average mutual information from slot 1 to $\mathrm{n}$ is decreasing with $n$ as a result of reduction in $I\left(S_{l} ; V_{l}\right)$ by increasing $l$. Moreover, the spiking probability that maximizes this average mutual information is decreasing with $n$. This optimum spiking probability and corresponding maximum mutual information from slot 1 to $n, C_{n}$, is achieved by simulations and plotted in Fig. 4(b) and Fig. 4(c). As it is shown in these figures, increasing $n$ decreases $C_{n}$ and corresponding optimum probability since average number of RRVs decrease as shown in Fig. 2, which in turn reduces the probability of successful bit transmission for $S_{n}=1$.

\section{Optimum Spiking Probability and Channel Capacity}

To evaluate the changes in the average mutual information when $n$ approaches $\infty$, i.e., $\lim _{n \rightarrow \infty} \sum_{l=1}^{n} \frac{I\left(S_{l} ; V_{l}\right)}{n}$, we evaluate the stationary probabilities with different numbers of RRVs and derive $I_{\infty}$, which is shown in Fig. 5(a). Moreover, the spiking probability and rate that maximizes $I_{\infty}$ for channels with different capacities of RP, $N_{\max }$, and the corresponding channel capacities, $C$, are shown in Fig. 5(b) and Fig. 5(c). Both optimum spiking probability and capacity of the channel increase with increasing $N_{\max }$ as illustrated in Fig. 5(b). To investigate the reason of this behavior, successful bit transmission probabilities are evaluated in Fig. 5(d). The bit error rate when there is no spike as input is almost zero, and the 


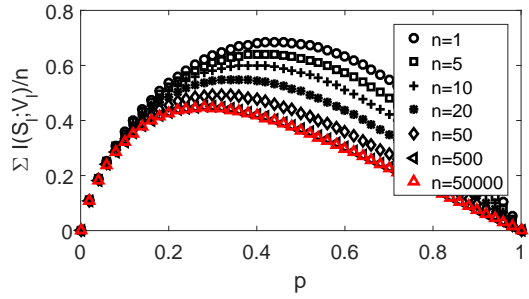

(a) Average mutual information for $N_{\max }=10$

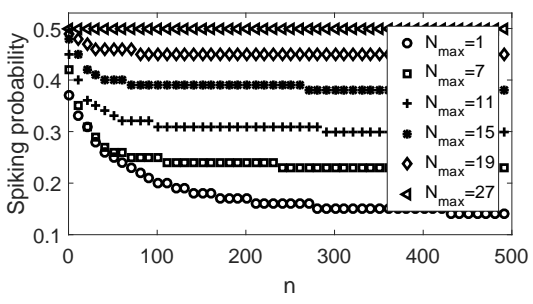

(b) Spiking probability that maximizes average mutual information from slot 1 to $n$

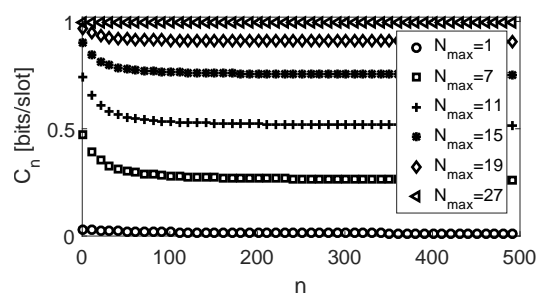

(c) Maximum mutual information

Fig. 4. Investigating maximum mutual information obtained by channel from slot 1 to $n$.

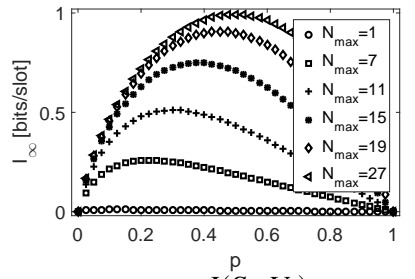

(a) $\lim _{n \rightarrow \infty} \sum_{l=1}^{n} \frac{I\left(S_{l} ; V_{l}\right)}{n}=I_{\infty}$

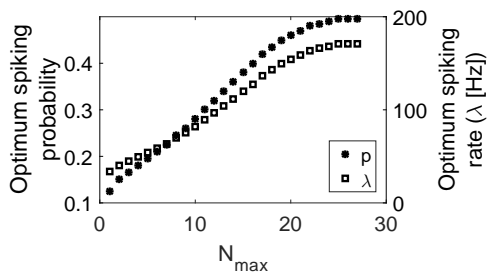

(b) Optimum spiking probability and rate

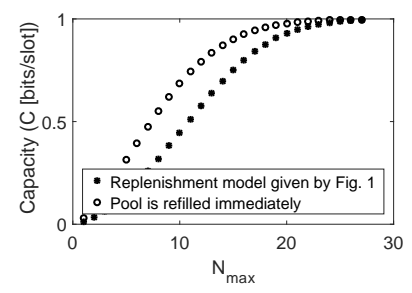

(c) Channel capacity

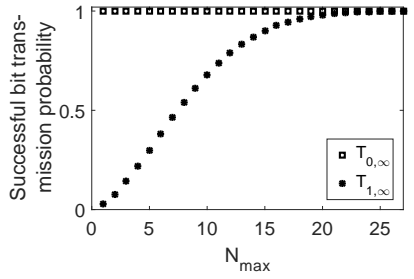

(d) Successful bit transmission probability

Fig. 5. Analyzing mutual information of the channel when $n$ approaches $\infty$, i.e., $I_{\infty}$, optimum spiking probability and channel capacity, i.e., C.

evoked release probability is much smaller than 1 for lower values of $N_{\max }$. Hence, the maximum mutual information is achieved with lower spiking probabilities when $N_{\max }$ is small. Moreover, successful bit transmission probabilities become equal for higher capacities of RP, which causes the optimum spiking probability and channel capacity to reach 0.5 and 1bit/slot as shown in Fig. 5(b) and Fig. 5(c), respectively.

The capacity derived by considering immediate replenishment of released vesicles is also shown in Fig. 5(c), which implies the impacts of inaccuracy in number of available vesicles for release on the capacity of vesicle release process. According to Fig. 5(c), existing studies in the literature overestimate the achievable rate of neuro-spike communication channel since none of them considers the changes in the vesicle release probability as a result of variations in the number of available vesicles for release [6]-[8].

The average RP capacity in Hippocampal neurons is 10 [14] for which utilizing $\lambda=82.13 \mathrm{~Hz}$ leads to achieve capacity of transmitting $0.44 \mathrm{bit} / \mathrm{slot}=110 \mathrm{bit} / \mathrm{s}$ information through vesicle release process as shown in Fig. 5(b) and Fig. 5(c).

\section{CONCLUSION}

In this paper, we studied a realistic communication model for vesicle release process and derived the mutual information between input and output of the channel for different time slots. We observed that this mutual information is decreasing with time as a result of ready pool depletion. Then, we calculated the maximum mutual information from slot 1 to $\mathrm{n}$ and its corresponding spiking probability. Finally, we derived the information capacity of vesicle release process and evaluated impacts of availability of vesicles on this capacity.

\section{ACKNOWLEDGMENT}

This work was supported in part by ERC project MINERVA (ERC-2013-CoG \#616922), EU project CIRCLE (EU-H2020-
FET-Open \#665564), and TŬBITAK graduate scholarship program (BIDEB-2215).

\section{REFERENCES}

[1] O. B. Akan et al., "Fundamentals of molecular information and communication science," Proc. IEEE, vol. 105, no. 2, pp. 306-318, 2017.

[2] H. Ramezani and O. B. Akan, "A communication theoretical modeling of axonal propagation in hippocampal pyramidal neuron," IEEE Trans. on NanoBiosci., to be published.

[3] E. Balevi and O. B. Akan, "A physical channel model for nanoscale neuro-spike communications," IEEE Trans. Commun., vol. 61, no. 3 , pp. 1178-1187, 2013.

[4] M. Veletić et al., "Peer-to-peer communication in neuronal nanonetwork," IEEE Trans. Commun., vol. 64, no. 3, pp. 1153-1166, 2016.

[5] H. Ramezani and O. B. Akan, "Synaptic channel model including effects of spike width variation," in Proc. of ACM NANOCOM'15, 2015, p. 11.

[6] D. Malak and O. B. Akan, "A communication theoretical analysis of synaptic multiple-access channel in hippocampal-cortical neurons," IEEE Trans. commun., vol. 61, no. 6, pp. 2457-2467, 2013

[7] A. Manwani and C. Koch, "Detecting and estimating signals over noisy and unreliable synapses: information-theoretic analysis," Neural computation, vol. 13, no. 1, pp. 1-33, 2001.

[8] M. Veletić et al., "On the upper bound of the information capacity in neuronal synapses," IEEE Trans Commun., vol. 64, no. 12, pp. 50255036, 2016.

[9] V. Matveev and X.-J. Wang, "Implications of all-or-none synaptic transmission and short-term depression beyond vesicle depletion: a computational study," J. Neurosci., vol. 20, no. 4, pp. 1575-1588, 2000.

[10] M. F. Bear et al., Neuroscience: Exploring the Brain, 3rd ed. Lippincott Williams \& Wilkins, 2007.

[11] A. A. Alabi and R. W. Tsien, "Synaptic vesicle pools and dynamics," Cold Spring Harbor perspectives in biology, vol. 4, no. 8, 2012.

[12] J. De La Rocha and N. Parga, "Short-term synaptic depression causes a non-monotonic response to correlated stimuli," J. Neurosci., vol. 25, no. 37, pp. 8416-8431, 2005.

[13] V. N. Murthy and C. F. Stevens, "Reversal of synaptic vesicle docking at central synapses," Nature neuroscience, vol. 2, pp. 503-507, 1999.

[14] T. Schikorski and C. F. Stevens, "Quantitative ultrastructural analysis of hippocampal excitatory synapses," J. Neurosci., vol. 17, no. 15, pp. 5858-5867, 1997

[15] L. E. Dobrunz and C. F. Stevens, "Heterogeneity of release probability, facilitation, and depletion at central synapses," Neuron, vol. 18, no. 6, pp. 995-1008, 1997. 